\title{
Urinary Obstruction, Stone Disease, and Infection
}

\author{
Refky Nicola and Christine O. Menias
}

\section{Learning Objectives}

- To review the pathogenesis of urinary infection, obstruction, and stones

- To discuss the imaging features of acute urologic infections such as renal abscess, acute pyelonephritis, and emphysematous pyelonephritis or chronic infections such as renal tuberculosis

- To illustrate the imaging of struvite or infection stones as well as highlight their complications

\subsection{Urinary Infection}

\subsubsection{Introduction}

Urinary tract infection is the most common urologic ailment, reported to affect 150 million patients annually worldwide. The diagnosis is usually based on clinical symptoms and urinalysis. In general, imaging is not necessary for the diagnosis of uncomplicated urinary tract infections in adult patients. However, diagnostic imaging can play an important role in the evaluation of patient's infections who do not respond to

R. Nicola, M.Sc. D.O.

Division of Abdominal Imaging, SUNY-Upstate Medical Center, Syracuse, NY, USA

C. O. Menias, M.D. (四)

Department of Radiology, Mayo Clinic in Arizona, Scottsdale, AZ, USA

e-mail: Menias.Christine@mayo.edu antibiotic treatment in the first $72 \mathrm{~h}$. It also helps to look for structural abnormalities of the urinary tract, which guide appropriate medical or surgical therapy. Various imaging modalities, including ultrasound (US), computed tomography $(\mathrm{CT})$, and magnetic resonance imaging (MRI), are useful in the diagnosis and depiction of urinary tract infections. This section highlights the imaging features of acute pyelonephritis, renal and perinephric abscess, pyonephrosis, emphysematous pyelonephritis, xanthogranulomatous pyelonephritis, and renal tuberculosis, using multimodality approach.

\subsubsection{Ultrasound}

Ultrasound (US) is a readily available imaging modality to serve as the initial modality in the evaluation of patients who have the clinical diagnosis of pyelonephritis. US serves as a good screening modality to evaluate for structural anatomic anomalies or complications of urinary tract infection, such as hydronephrosis and obstructive uropathy. Gray-scale imaging features of pyelonephritis include renal enlargement, hydronephrosis, loss of renal sinus fat due to edema, loss of corticomedullary differentiation, areas of hypoperfusion on color Doppler, and abscess formation [1]. There may be changes in the renal echogenic pattern and the involved kidney which can be either hyperechoic or hypoechoic. The urinary bladder may also be evaluated in this setting and can show amount of residual urine volume, presence of bladder wall thickening, or potentially a stone. However, ultrasound is limited in its ability to differentiate calcification from gas within the collecting system or parenchyma, visualization of small abscesses, and perinephric extension of infection which are all common in early infections. In one study, ultrasound findings were noted in only $24 \%$ of patients, while other studies report 
only $20 \%$ of patients [2, 3]. However, new techniques such as tissue harmonics have showed sensitivity and specificity of $97 \%$ and $80 \%$ [3].

\subsubsection{CT Technique}

CT is the preferred imaging modality for the evaluation of complicated pyelonephritis because it provides both physiologic and anatomic detail and identifies both extrarenal and intrarenal pathology [4]. An unenhanced CT is excellent for identifying gas in the urinary tract, stones, hemorrhage, and dilatation of the urinary tract [5]. Regions of higher attenuation on non-contrast CT are associated with hemorrhage, while areas of lower attenuation represent edema. Following intravenous contrast administration, pyelonephritis manifests as "wedge shaped" or linear regions of decreased enhancement that spans from the renal sinus to the renal cortex. The excretory phase, obtained between 3 and 5 min after the administration of contrast, should be performed in suspected urinary obstruction [2]. CT also can evaluate for additional features of renal inflammation and its complications, including inflammation of the perinephric fat, thickening of Gerota's fascia, and abscess formation.

\subsubsection{Acute Renal Infection}

\subsubsection{Acute Pyelonephritis}

CT is indicated when complications of renal infection are suspected. Predisposing conditions, including urinary calculi, neurogenic bladder, immune system compromise, diabetes, IV drug abuse, or chronic debilitating disease, increase the risk of complications that require intervention. Most urinary tract infections are caused by gram-negative bacilli, but the incidence of fungal and tuberculous infections is rising. Acute pyelonephritis is a multifocal infection of one or both kidneys, which can result from either hematogenous spread or from ascending infection from the bladder and usually resolves after appropriate antibiotic therapy. Adults who fail to improve should be imaged to detect complications.

CT signs of acute bacterial infection of the kidneys include wedge-shaped areas of decreased parenchymal enhancement due to decreased blood flow caused by edema. A striated pattern of linear alternating increased and decreased attenuation ("striated nephrogram") on enhanced scans is classic [6]. This differential enhancement is contributed by various factors, namely, tubular obstruction by inflammatory debris, interstitial edema, and vasospasm which tend to decrease the flow of contrast through tubules [7, 8].

\subsubsection{Acute Renal/Perirenal Abscess}

Untreated or inadequately treated pyelonephritis can lead to suppuration, liquefaction, and parenchymal necrosis that eventually leads to abscess. Patients at risk of abscess formation include those with diabetes, chronic diseases, immunocompromised state, urinary tract obstruction, and intravenous drug abusers. Renal abscesses tend to be solitary and may decompress into the perinephric space or collecting system. On US, they usually are hypoechoic with through transmission, mobile debris, and occasional air pockets and on color Doppler lack internal flow. US is less sensitive in determining the presence and extent of perinephric extension relative to CT; however, it is an appropriate modality for follow-up imaging to look for response to treatment and as a guide for percutaneous drainage. CT demonstrates a fluid collection with an enhancing rim which may contain gas. Large abscesses usually require catheter or surgical drainage (Fig. 20.1). Extension of infection into the perirenal space is common, and perirenal abscesses may develop. Extra-parenchymal collections can extend to the psoas muscle, pelvis, or groin $[4,6]$.

\subsubsection{Emphysematous Pyelonephritis}

Emphysematous pyelonephritis is a severe life-threatening necrotizing type of pyelonephritis that develops in mostly diabetics $(90 \%)$, but also in immunocompromised patients,

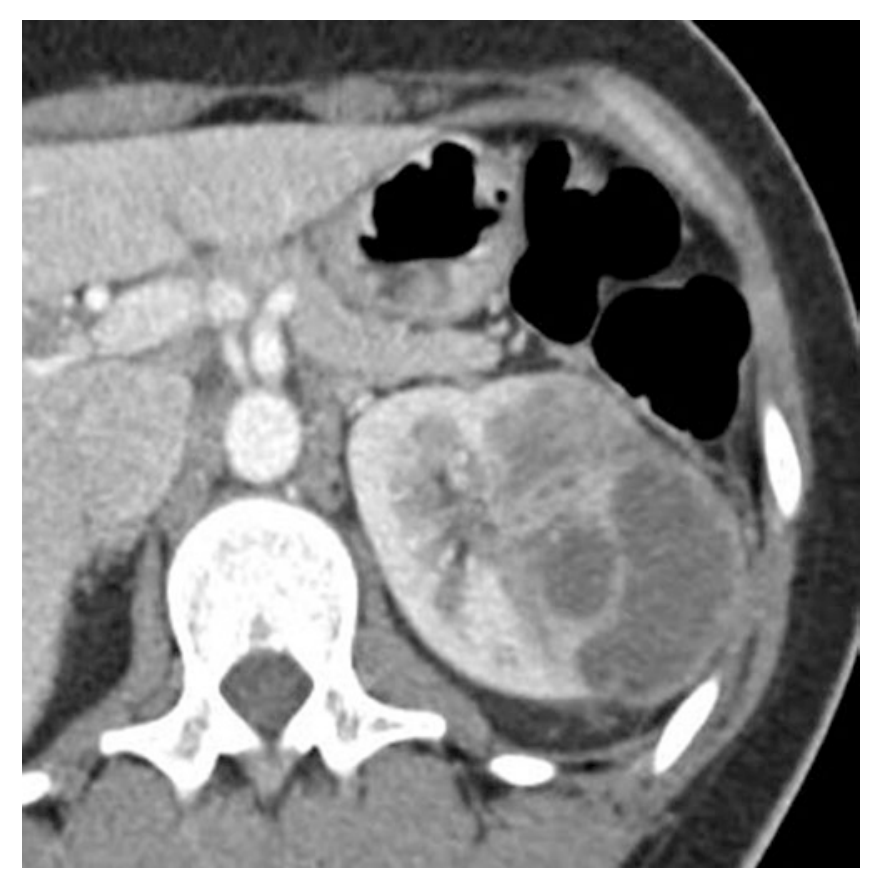

Fig. 20.1 Renal Abscess in a 37-year-old female with HIV and fever. Axial contrast-enhanced CT image of the left kidney shows a complex peripherally enhancing fluid collection and associated perinephric stranding. Subsequent drained fluid grew Staphylococcus aureus 
and patients with urinary obstruction. Gas is produced from metabolism of glucose by gram-negative bacteria. CT shows gas in the renal parenchyma in addition to signs of renal inflammation. The most common gas-forming organisms are E. coli, Klebsiella pneumoniae, and Proteus mirabilis which are also the same organisms associated with struvite stones. US shows an enlarged kidney with nondependent echoes (gas) in the parenchyma or collecting system, with "dirty shadowing" that helps differentiate from stones. US is limited in evaluating the extent of the disease. CT is the imaging modality of choice for emphysematous pyelonephritis with findings of parenchymal enlargement and destruction without or with abscess formation, small streaks of gas, or fluid collections [4, 9, 10].

Emphysematous pyelonephritis is classified into two types based on imaging features and prognosis.

Type 1 emphysematous pyelonephritis is renal parenchymal destruction with streaky or mottled areas of gas without an extrarenal fluid collection. Type 2 is renal or perirenal fluid collections that are directly associated with a loculated gas or gas within the collecting system. The mortality for type 1 and type 2 is $69 \%$ and $18 \%$, respectively.

\subsubsection{Pyonephrosis}

Pyonephrosis is an acute infection with pus within an obstructed collecting system. Renal destruction is rapid, and urgent drainage of the collecting system is required. The obstruction can be due to tumor, stricture, sloughed papilla, tumor, or stones. If patients are left untreated, patients may develop loss of renal function and septic shock [11, 12]. US features include dilatation of the pelvicalyceal system, internal collecting system fluid-fluid levels, echogenic debris, and occasionally dirty echoes of gas. CT features may show a dilated but thick-walled collecting system, distended with high attenuation pus-filled fluid, and thinning of the renal cortex, and typically source of obstruction.

\subsubsection{Renal Tuberculosis}

Tuberculosis (TB) remains the leading global cause of death from infectious disease [4]. The urinary tract is the most common extra-pulmonary site of infection and results from hematogenous dissemination in $15-20 \%$ of cases [4]. The rise of tuberculosis within developed countries is caused by the spread of HIV (human immunodeficiency viral) infection and antibiotic-resistant strains of mycobacterium. The urinary tract may be involved even though chest radiographs do not show evidence of TB. Multiple caseous granulomas form in the cortex because of its favorable blood supply. These may remain dormant or reactivate, spreading organisms to the tubules, resulting in papillary necrosis. Progressive infection will eventually destroy the kidney. The common imaging findings of renal tuberculosis are due to papillary necrosis which is followed by parenchymal destruction. Parenchymal lesions lead to corticomedullary scarring and renal contour scarring. Pelvoinfundibular stricture, cortical low-attenuation lesions, scarring, and papillary necrosis and calcifications are findings which are all associated with renal tuberculosis $[13,14]$. Calcification is very commonly seen and varies from granular, amorphous, and curvilinear which can replace segments or the entire renal parenchyma resulting in the end stage "putty kidney" [14].

\subsection{Urinary Stones and Obstruction}

\subsubsection{Introduction}

The most common cause of urinary obstruction in the emergent setting is urolithiasis, but chronic urinary obstruction has multiple etiologies such as lymphadenopathy or retroperitoneal fibrosis. Also, the patients with a history of urothelial, cervical, prostate, or colon carcinoma are also susceptible to chronic urinary obstruction due to neoplastic obstruction [15]. Therefore, the role of the radiologist is pivotal in differentiating between acute and chronic urinary obstruction because an accurate diagnosis is essential in avoiding serious complications such as sepsis or chronic renal insufficiency.

\subsubsection{Epidemiology}

Urolithiasis has become a problem which transcends geography, culture, and gender. Approximately, 1.2 million Americans are affected each year. About $12 \%$ of men and $5 \%$ of women will develop urolithiasis during their lifetime. However, the prevalence is estimated to be higher in more developed countries [16-20]. Stone disease is also one of America's costliest health issues, accounting for approximately \$2 billion annual health-care dollars [21].

\subsubsection{Stone Composition}

There are several dietary, hereditary, and environmental factors which play an integral role in stone disease, such as hypercalciuria, renal tubular acidosis, and chronic diarrhea and gout [20]. The composition of most upper urinary tract stones is either calcium (i.e., calcium oxalate dehydrate, calcium oxalate monohydrate, calcium phosphate), struvite, or uric acid with calcium being the most common. Less common compositions include protein matrix, cystine, brushite, or stones due 
to drug-related therapies such as indinavir. Even though most stones are visible on CT, the struvite stones and uric acid stones are radiolucent on radiography [22]. Due to the difference in composition of renal stones, there is a wide variation in the attenuation on multidetector CT. For example, calcium monohydrate and brushite measure 1700-2800 HU, calcium phosphate 1200-1600 HU, cystine 600-1100 HU, struvite 600-900 HU, and uric acid 200-450 HU [23-25].

The importance of identifying the stone composition is directly related to management. Uric acid stones are managed with medical therapy. Cystine- and calcium-based stones cannot be easily fragmented with shock wave lithotripsy (SWL) [22].

\subsubsection{Clinical Perspective}

The most common complaint in patients with urolithiasis is acute flank pain which is eventually relieved with the passage of the stone. If the stone is lodged at the ureteropelvic junction (UPJ), the pain will radiate to the groin. However, if the stone is located distally at the ureterovesical junction (UVJ), it will cause suprapubic discomfort and voiding urgency. Pain typically radiates to the testis or labium [26]. In addition, patients will complain of vomiting, nausea, and microscopic hematuria. However, staghorn calculus which is caused by urease-producing bacteria such Proteus mirabilis, Pseudomonas aeruginosa, Klebsiella pneumoniae, and E. coli can manifest as a recurrent urinary tract infection instead of an obstruction [20]. If there is an infection and an obstructed collecting system, patients can present with sepsis [27]. Microscopic or macroscopic hematuria can occur regardless of urinary obstruction. Usually, a urinalysis and complete blood count is done to evaluate for infection.

\subsubsection{CT Technique}

Since 1995 unenhanced MDCT is the primary modality of choice in the evaluation of patients with renal colic and accounts for $20 \%$ of all CT examinations which are performed for acute abdominal pain. Thin (1-3 $\mathrm{mm}$ ) reconstruction sections are helpful for detection of small stones. However, $5 \mathrm{~mm}$ MDCT with $3 \mathrm{~mm}$ coronal reformatted images has been found to improve stone detection while maintaining radiation dose benefits [28]. A tube potential of $100-120 \mathrm{kvp}$ and automatic tube current modulation is performed with a tube current of 80 to $500 \mathrm{mAs}$. Typically, intravenous contrast administration is not required for the evaluation of renal calculi at CT.

Unlike MDCT which uses a single energy source, dualenergy CT (DECT) allows for acquisition of CT images at two different energies. With MDCT, stone composition is difficult to assess since there is significant overlap in attenu- ation for each of the stone subtypes. By comparing the attenuation of a stone at two different energy levels, the mystery of the stone's composition is somewhat mitigated. For example, this becomes particularly useful in differentiating uric acid from calcium-based stones, since both have similar attenuation coefficient on single-energy CT [26].

\subsubsection{Struvite Stone and their Complications}

Struvite stones, which are also known as infection stones, are composed of magnesium ammonium phosphate and comprise $15-20 \%$ of all renal calculi. Struvite stones develop more frequently in a patient with urinary stasis or recurrent urinary tract infections. Struvite stones with superimposed pyelonephritis are caused by bacteria entering the renal tubules and inciting an inflammatory response which involves the tubules and renal interstitium [7]. CT is useful in assessing intra- and extrarenal inflammation such as gas, calculus, and hemorrhage [4]. CT shows the inciting staghorn calculus with superimposed pyelonephritis with wedgeshaped areas of decreased enhancement corresponding to the striated nephrogram and perinephric stranding [7, 8]. Another complication is pyonephrosis, which is a dilated, obstructed, and infected collecting system. Struvite calculi can obstruct the pelvicalyceal system with superimposed infection and can lead to significant morbidity if not adequately treated.

\subsubsection{Xanthogranulomatous Pyelonephritis}

Xanthogranulomatous pyelonephritis is a rare form of chronic pyelonephritis which develops from chronic obstruction due to struvite/infected renal stones resulting in a chronic granulomatous inflammatory response and eventual destruction and replacement of the renal parenchyma, by lipid-laden macrophages. The most common inciting organisms are E. coli and Proteus mirabilis which are organisms associated with struvite/infection stones. XGPN classically presents in mid-aged female patients with recurrent urinary tract infection, diabetes, or obstructing urinary stones [2, 5]. XGPN may involve the entire kidney (diffuse) or related to an obstructed upper or lower pole (focal), which at times is difficult to differentiate from tumor or abscess.

\subsubsection{Imaging of XGPN}

US features of XGPN are nonspecific and include focal or diffuse enlargement of the kidney with renal cortical thinning, and echogenicity within the calyces, and typically a large UPJ stone with posterior acoustic shadowing due to 


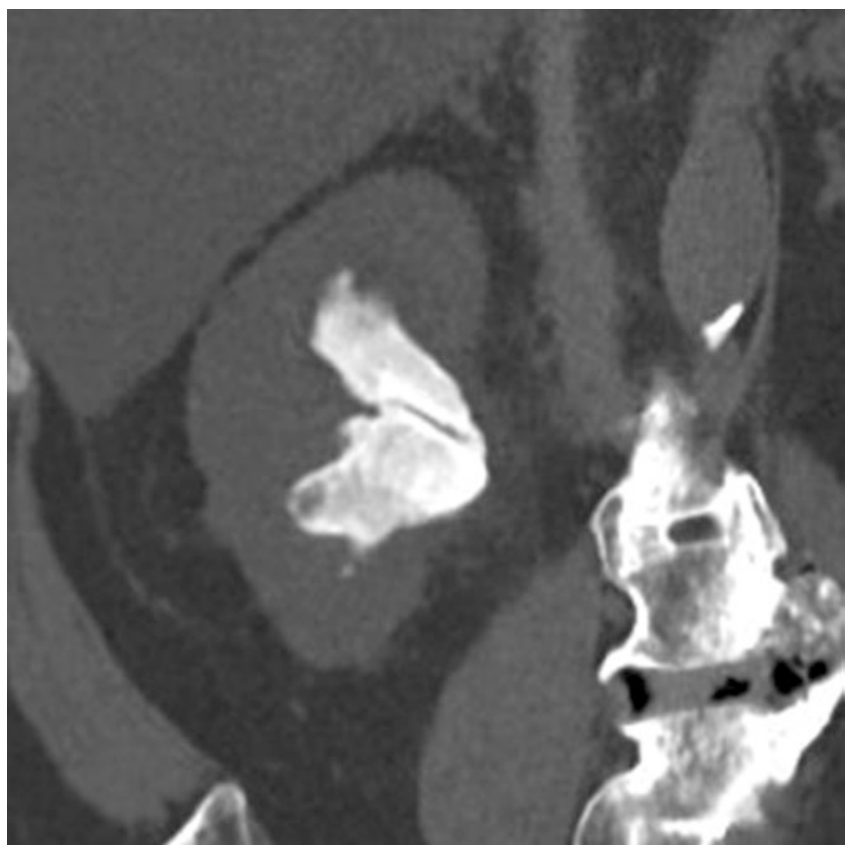

Fig. 20.2 XGPN in a 56-year-old female with flank pain and urinary tract infection. Axial contrast-enhanced CT image shows an enlarged non-functioning left kidney with blown-out calyces and "bear paw" appearance, with a large central staghorn struvite/infection stone. Subsequent nephrectomy specimen confirmed XGPN

the stone, obscuring detail of the kidney. Contrast-enhanced $\mathrm{CT}$ is the modality of choice in the evaluation of XGPN; however, MR serves as a useful alternative when IV contrast cannot be administered. CT may show an enlarged kidney replaced with low-attenuation dilated/blown-out calyces resulting in renal cortical thinning, resulting in the classic "bear paw sign" [4]. These blown-out calyces are thought to represent dilated calyces filled with hemorrhage, debris, or pus (Fig. 20.2). XGPN results in a non-functioning enlarged kidney, with typically a large central staghorn/infected struvite stone that fails to excrete on the excretory phase [4] (Fig. 20.3). Extrarenal extension of XGPN is often present with inflammatory changes and abscess that track from the kidney and dissect through the posterior flank, psoas muscle, and adjacent viscera like the liver and spleen.

\subsubsection{Renal Replacement Lipomatosis}

Another end-spectrum complication of long-standing stone disease is complete destruction and atrophy of the renal sinus, perinephric space, and hilum with subsequent fatty replacement, known as renal sinus or replacement lipomatosis (RRL) [29]. In the most extensive form of RRL, there is total atrophy of the renal parenchyma and complete fibrofatty replacement. RRL is typically unilateral and linked to renal stones in 76-79\% and results from severe renal atrophy or destruction due to chronic stone disease, chronic pyelone-

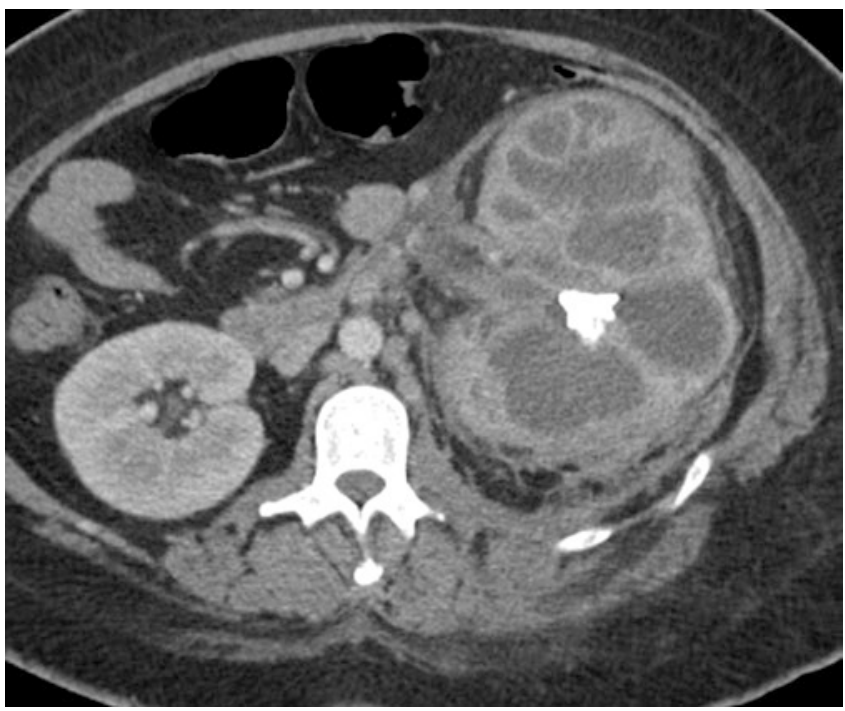

Fig. 20.3 Staghorn calculus in a 68-year-old female with flank pain. Coronal non-contrast CT image in bone windows shows a large stone filling in the right renal pelvocalyceal system with a staghorn appearance consistent with an infection stone. Stone analysis subsequently showed struvite/magnesium ammonium phosphate. Note the subtle perinephric inflammatory stranding

phritis, and renal tuberculosis [29]. US findings may demonstrate a large hyperechoic fatty mass which may suggest the diagnosis; however, parenchymal and renal sinus fat deposition may not be well visualized on ultrasound, and the echogenicity of fat may obscure detection of its associated stone. CT is the modality of choice, to confirm the end stage renal atrophy and fatty proliferation in the renal sinus and perinephric space, and helps differentiate RRL from fatcontaining neoplasms.

\subsection{Concluding Remarks}

Ultrasound and CT play an important role in the evaluation of patients who present with acute urinary tract infection, obstruction, and stone disease. Familiarity with the pathogenesis, imaging features, and treatment of these entities can aid radiologic diagnoses and guide appropriate patient management.

\section{Take-Home Message}

- Ultrasound and CT play an important role in the evaluation of patients who present with acute urinary tract infection, obstruction, and stone disease. Familiarity with the pathogenesis, imaging features, and treatment of these entities can aid radiologic diagnoses and guide appropriate patient management. 


\section{References}

1. Rigsby CM, Rosenfield AT, Glickman MG, Hodson J. Hemorrhagic focal bacterial nephritis: findings on gray-scale sonography and CT. AJR Am J Roentgenol. 1986;146(6):1173-7. https://doi. org/10.2214/ajr.146.6.1173.

2. Stunell H, Buckley O, Feeney J, Geoghegan T, Browne RF, Torreggiani WC. Imaging of acute pyelonephritis in the adult. Eur Radiol. 2007;17(7):1820-8. https://doi.org/10.1007/ s00330-006-0366-3.

3. Kim B, Lim HK, Choi MH, Woo JY, Ryu J, Kim S, Peck KR. Detection of parenchymal abnormalities in acute pyelonephritis by pulse inversion harmonic imaging with or without microbubble ultrasonographic contrast agent: correlation with computed tomography. J Ultrasound Med. 2001;20(1):5-14.

4. Craig WD, Wagner BJ, Travis MD. Pyelonephritis: radiologicpathologic review. Radiographics. 2008;28(1):255-77; quiz 327258. https://doi.org/10.1148/rg.281075171.

5. Goldman SM, Fishman EK. Upper urinary tract infection: the current role of CT, ultrasound, and MRI. Semin Ultrasound CT MR. 1991;12(4):335-60.

6. Kawashima A, Sandler CM, Goldman SM, Raval BK, Fishman EK. CT of renal inflammatory disease. Radiographics. 1997;17(4):851-66; discussion 867-858. https://doi.org/10.1148/ radiographics.17.4.9225387.

7. Talner LB, Davidson AJ, Lebowitz RL, Dalla Palma L, Goldman SM. Acute pyelonephritis: can we agree on terminology? Radiology. 1994;192(2):297-305. https://doi.org/10.1148/ radiology.192.2.8029384.

8. Dalla-Palma L, Pozzi-Mucelli F, Pozzi-Mucelli RS. Delayed CT findings in acute renal infection. Clin Radiol. 1995;50(6):364-70.

9. Silver TM, Kass EJ, Thornbury JR, Konnak JW, Wolfman MG. The radiological spectrum of acute pyelonephritis in adults and adolescents. Radiology. 1976;118(1):65-71. https://doi. org/10.1148/118.1.65.

10. Wan YL, Lee TY, Bullard MJ, Tsai CC. Acute gas-producing bacterial renal infection: correlation between imaging findings and clinical outcome. Radiology. 1996;198(2):433-8. https://doi. org/10.1148/radiology.198.2.8596845.

11. Yoder IC, Pfister RC, Lindfors KK, Newhouse JH. Pyonephrosis: imaging and intervention. AJR Am J Roentgenol. 1983;141(4):73540. https://doi.org/10.2214/ajr.141.4.735.

12. Subramanyam BR, Raghavendra BN, Bosniak MA, Lefleur RS, Rosen RJ, Horii SC. Sonography of pyonephrosis: a prospective study. AJR Am J Roentgenol. 1983;140(5):991-3. https://doi. org/10.2214/ajr.140.5.991.

13. Kenney PJ. Imaging of chronic renal infections. AJR Am J Roentgenol. 1990;155(3):485-94. https://doi.org/10.2214/ ajr.155.3.2117344.

14. Muttarak M, ChiangMai WN, Lojanapiwat B. Tuberculosis of the genitourinary tract: imaging features with pathological correlation. Singap Med J. 2005;46(10):568-74; quiz 575.
15. Farrell TA, Hicks ME. A review of radiologically guided percutaneous nephrostomies in 303 patients. J Vasc Interv Radiol. 1997;8(5):769-74.

16. Curhan GC. Epidemiology of stone disease. Urol Clin North Am. 2007;34(3):287-93. https://doi.org/10.1016/j.ucl.2007.04.003.

17. Stamatelou KK, Francis ME, Jones CA, Nyberg LM, Curhan GC. Time trends in reported prevalence of kidney stones in the United States: 1976-1994. Kidney Int. 2003;63(5):1817-23. https://doi.org/10.1046/j.1523-1755.2003.00917.x.

18. Johnson CM, Wilson DM, O'Fallon WM, Malek RS, Kurland LT. Renal stone epidemiology: a 25-year study in Rochester, Minnesota. Kidney Int. 1979;16(5):624-31.

19. Soucie JM, Thun MJ, Coates RJ, McClellan W, Austin $\mathrm{H}$. Demographic and geographic variability of kidney stones in the United States. Kidney Int. 1994;46(3):893-9.

20. Cheng PM, Moin P, Dunn MD, Boswell WD, Duddalwar VA. What the radiologist needs to know about urolithiasis: part 1-pathogenesis, types, assessment, and variant anatomy. AJR Am J Roentgenol. 2012;198(6):W540-7. https://doi.org/10.2214/AJR.10.7285.

21. Pearle MS, Calhoun EA, Curhan GC, Urologic Diseases of America P. Urologic diseases in America project: urolithiasis. J Urol. 2005;173(3):848-57. https://doi.org/10.1097/01. ju.0000152082.14384.d7.

22. Kambadakone AR, Eisner BH, Catalano OA, Sahani DV. New and evolving concepts in the imaging and management of urolithiasis: urologists' perspective. Radiographics. 2010;30(3):603-23. https:// doi.org/10.1148/rg.303095146.

23. Saw KC, McAteer JA, Monga AG, Chua GT, Lingeman JE, Williams JC Jr. Helical CT of urinary calculi: effect of stone composition, stone size, and scan collimation. AJR Am J Roentgenol. 2000;175(2):329-32. https://doi.org/10.2214/ajr.175.2.1750329.

24. Bellin MF, Renard-Penna R, Conort P, Bissery A, Meric JB, Daudon M, Mallet A, Richard F, Grenier P. Helical CT evaluation of the chemical composition of urinary tract calculi with a discriminant analysis of CT-attenuation values and density. Eur Radiol. 2004;14(11):2134-40. https://doi.org/10.1007/s00330-004-2365-6.

25. Mostafavi MR, Ernst RD, Saltzman B. Accurate determination of chemical composition of urinary calculi by spiral computerized tomography. J Urol. 1998;159(3):673-5.

26. Masch WR, Cronin KC, Sahani DV, Kambadakone A. Imaging in Urolithiasis. Radiol Clin N Am. 2017;55(2):209-24. https://doi. org/10.1016/j.rcl.2016.10.002.

27. Conrad S, Busch R, Huland H. Complicated urinary tract infections. Eur Urol. 1991;19(Suppl 1):16-22.

28. Lin WC, Uppot RN, Li CS, Hahn PF, Sahani DV. Value of automated coronal reformations from 64-section multidetector row computerized tomography in the diagnosis of urinary stone disease. J Urol. 2007;178(3 Pt 1):907-11. https://doi.org/10.1016/j. juro.2007.05.042; discussion 911.

29. Chang SD, Coakley FV, Goldstein RB. Case report: renal replacement lipomatosis associated with renal transplantation. Br J Radiol. 2005;78(925):60-1. https://doi.org/10.1259/bjr/31723131.

Open Access This chapter is licensed under the terms of the Creative Commons Attribution 4.0 International License (http://creativecommons. org/licenses/by/4.0/), which permits use, sharing, adaptation, distribution and reproduction in any medium or format, as long as you give appropriate credit to the original author(s) and the source, provide a link to the Creative Commons license and indicate if changes were made.

The images or other third party material in this book are included in the book's Creative Commons license, unless indicated otherwise in a credit line to the material. If material is not included in the book's Creative Commons license and your intended use is not permitted by statutory regulation or exceeds the permitted use, you will need to obtain permission directly from the copyright holder. 